\title{
Dystonia in central pontine myelinolysis without evidence of extrapontine myelinolysis
}

\author{
Stephen M Salerno, Roger Kurlan, Stephen E Joy, Ira Shoulson
}

\begin{abstract}
A 44-year-old female is described who developed persistent upper extremity and orolingual dystonia several weeks after suspected onset of central pontine myelinolysis (CPM), later confirmed by characteristic pontine lesions on MRI. No foci of the extrapontine myelinolysis were evident. This case confirms that dystonia may be a late and persistent sequela of CPM and may occur in the absence of visible lesions outside the brainstem.
\end{abstract}

$(\mathcal{A}$ Neurol Neurosurg Psychiatry 1993;56:1221-1223)

Central pontine myelinolysis (CPM) is an acute demyelinating disorder involving the ventral pons and is usually precipated by rapid fluctuations in serum electrolytes. The antemortem diagnosis of CPM has been made more reliable with the introduction of CPM are related to the characteristic pontine location of the demyelination. These include dysarthria, dysphagia, emotional incontinence, impaired consciousness, and quadraparesis. Movement disorders are not usual sequelae of CPM and in only a very few cases has dystonia been described. ${ }^{1-4}$ Based on MRI findings, Dickoff et al suggested that movement disorders occurring with CPM are the result of extrapontine myelinolysis (EPM) involving the basal ganglia. ${ }^{5}$ We report a patient who developed persistent dystonic features with MRI documented pontine lesions indicative of CPM but no radiologically evident foci of demyelination outside the brainstem.

Neurology, University of Rochester School of Medicine, Rochester, New York, USA

$S$ M Salerno

R Kurlan

$S$ E Joy

I Shoulson

Correspondence to:

Dr Salerno, Department of

Neurology, University of

Rochester School of

Mechester School of, NY

14642 8673, USA

Received 17 July 1992 and

in final revised form

8 February 1993.

Accepted 11 February 1993

\section{Case report}

A 44-year-old woman with a history of hypertension, depression, and schizophrenia treated with perphenazine $4 \mathrm{mg}$ twice a day presented to a local emergency room complaining of vertigo, fatigue, and leg cramps. She had been started on metolazone and meclizine about one week before admission.

Physical examination showed a blood pressure of 150/110, but was otherwise normal. Serum chemistries demonstrated sodium 118 $\mathrm{mEq} / \mathrm{l}$, and mildly elevated liver enzymes. MRI. The most typical manifestations of

Over the next week she ate poorly, became lethargic, and then unresponsive with only slight movement to painful stimuli. All brainstem reflexes were intact. A repeat serum sodium was found to be $109 \mathrm{mEq} / \mathrm{l}$, and the patient received $5 \%$ saline infused at an unknown rate. Two days later her serum sodium was normal. The patient was then transferred to another hospital.

Cranial CT and lumbar puncture performed after transfer were normal and an electroencephalogram showed moderate slowing. The patient remained comatose with abnormal flexor posturing of all limbs. After two weeks, she became alert but remained unresponsive to commands and had no spontaneous movements, indicative of akinetic mutism. Over the next three months, she slowly improved and became able to walk and write. However, severe dystonia of both arms and the orolingual region developed. She was able to speak but was dysarthric. The patient was discharged four months after her initial admission.

Five months after discharge, she had episodes of uncontrollable crying and her severe dystonia persisted, particularly in the right arm. Cranial CT was again normal. She had electroconvulsive therapy for a suspected depressive disorder.

Thirteen months after her initial admission, the patient had left cryothalamotomy to relieve her marked right arm dystonia. The procedure produced no improvement. Over the next year, her speech deteriorated and she required a communication board. She had increasing dystonia of the left arm and developed truncal dystonia as well. Treatment with levodopa/carbidopa 10/100 4 times a day haloperidol $1 \mathrm{mg} 4$ times a day or lorazepam $2.5 \mathrm{mg}$ twice a day produced no benefit.

Thirty months after her initial admission, the patient was admitted to Strong Memorial Hospital. There was marked dystonia in the upper limbs, upper trunk, oropharynx, and tongue, largely sparing her lower trunk and legs. Reflexes were $2+$ and symmetrical and plantar reflexes were flexor. There was no sensory deficit. Cranial CT revealed mild atrophy of the brainstem and cerebellar vermis. A 1.5 tesla T1 weighted parasaggital cranial MRI demonstrated an oval focus of low signal within the mid-pons (fig A) and a 1.5 tesla T2 weighted axial MRI showed a 
Figure $A: 1 \cdot 5$ tesla $T 1$ weighted (PS; TR $400 \mathrm{ms,}$ TE $25 \mathrm{~ms}$ ) parasaggital cranial MRI

demonstrating an oval focus of decreased signal within the mid pons:

$B: 1 \cdot 5$ tesla $T 2$ weighted (SE; TR $2000 \mathrm{ms,TE}$ $25 \mathrm{~ms}$ ) axial cranial $M R I$ showing a lobulated band of increased signal centrally within the pons.

$P S=$ partial saturation

$S E=$ spin echo $T R=$

repetition time $T E=$ echo delay time
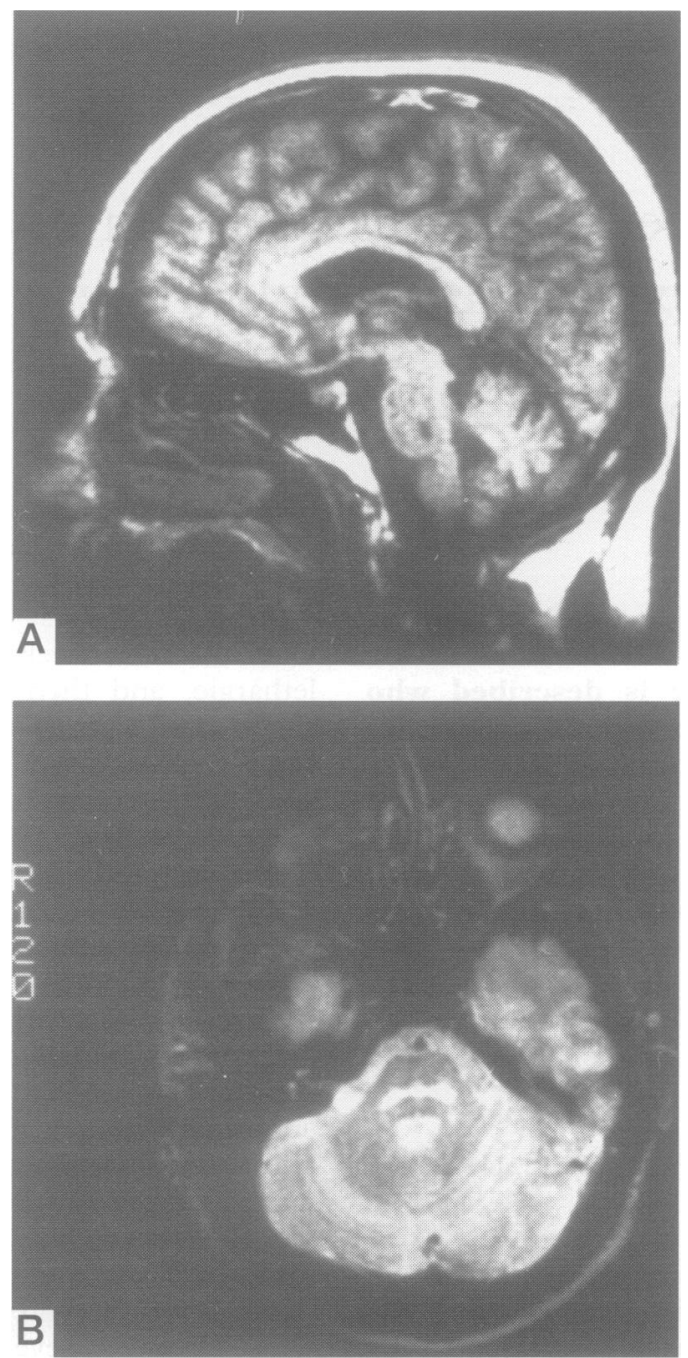

lobulated band of high signal centrally within the pons (fig B) indicative of CPM.

Clonazepam at $1.5 \mathrm{mg} /$ day produced mild improvement of speech and right arm mobility. Further dosage increases to $3 \mathrm{mg} /$ day did not add further benefit. Amitriptyline and baclofen were added without apparent benefit.

\section{Discussion}

This case represents an occurrence of persistent oropharyngeal, upper trunk, and upper limb dystonia after correction of severe hyponatraemia with hypertonic saline, a clinical setting known to be associated with the development of central pontine myelinolysis. The diagnosis of CPM is supported by clinical features of pontine dysfunction including quadraparesis, dysarthria, and emotional lability and characteristic MRI-demonstrated demyelinative lesions. Similar to other reported cases, ${ }^{1-4}$ dystonia occurred as a late sequela of CPM with onset several weeks after the acute illness. This patient showed no evidence of EPM, a possible explanation for movement disorders previously observed in CPM.

Other authors have reported movement disorders in patients with CPM without radiological evidence for EPM. Grafton et al described a patient who developed segmental dystonia of the right shoulder, arm, and neck four months after initial presentation with CPM. ${ }^{1}$ There was no MRI evidence of EPM. Thompson observed a patient who developed dystonic posturing of the upper limbs two years after onset of CPM, also in the absence of basal ganglia involvement by MRI. ${ }^{2}$ Tison et al examined a patient who developed generalised dystonia and choreoathetosis several weeks after her initial diagnosis of CPM. ${ }^{3}$ The symptoms progressed to a maximum over four months and then improved spontaneously. No basal ganglia lesions were present. On the other hand, Maraganore et al found radiographic evidence of EPM involving the basal ganglia in two patients with CPM who developed upper body dystonia. ${ }^{4}$

Thus the majority of reported cases of dystonia occurring with CPM have not had radiographic evidence of EPM. It remains unclear whether the dystonia is a result of undetected basal ganglia lesions, a manifestation of visible pontine pathology, or a combination of both. Evidence exists that demyelinative lesions in CPM may take as long as a month to manifest themselves radiographically, which might explain the apparent absence of EPM in some cases. ${ }^{6}$

Pontine lesions of other aetiologies can cause a variety of syndromes, but do not generally cause movement disorders unless associated with cerebellar lesions. ${ }^{7}$ However, CPM without evidence of EPM has caused dystonia, choreoathetosis, and Parkinsonism. Even though dystonia is characteristically associated with grey matter lesions, especially of the putamen, CPM is generally thought of as a white matter disease. ${ }^{8}$ White matter involvement causing dystonia is not unknown in other demylinative disorders such as multiple sclerosis. ${ }^{910}$ Postmortem examination confirming that demyelination really is confined to the pons would be helpful to clarify this matter.

Our experience suggests that the diagnosis of CPM should be suspected in patients who develop unexplained dystonic features particularly in the setting of known predisposing medical conditions such as hyponatraemia, malnutrition, and alcoholism. Dystonia occurring in association with CPM may occur with or without MRI demonstrated foci of EPM and it remains unclear whether the pontine lesion or an undetected focus of EPM is responsible.

Mrs Donna LaDonna prepared the manuscript. This work was supported in part by a Short-Term training gran (HL-07496) from the National Institutes of Health (to SS)

1 Grafton ST, Bahls F, Bell RK. Acquired dystonia following central pontine myelinolysis (abstract). Neurology 1987;37(suppl 1):276 (abstr)

2 Thompson PD, Miller D, Gledhill RF, Rossor MN. Magnetic resonance imaging in central pontine myelinolysis. ₹ Neurol Neurosurg Psychiatry 1989;52:675-7.

3 Tison FX, Ferrer X, Julien J. Delayed onset movemen disorders as a complication of central pontine myelinolysis. Mov Disord 1991;6:171-3. 
4 Maraganore DM, Folger WN, Swanson JW, Ahlskog JE. Movement disorders as sequelae of central pontine myelinolysis: report of three cases. Mov Disord 1992;7: 142-8.

5 Dickoff DJ, Raps M, Yahr MD. Striatal syndrome following hyponatremia and its rapid correction. A manifestation of extrapontine myelinolysis confirmed with tion of extrapontine myelinolysis confirmed with magne

6 Clifford DB, Gado MH, Levy BK. Osmotic demyelination syndrome. Lack of pathological and radiologic imaging correlation. Arch Neurol 1989;46: 343-7.
7 Brazis PW. The localization of lesions affecting the brainstem. In: Brazis PW, Masdea JC, Biller J, eds. Localization in clinical neurology. Boston: Little Brown, 1990:269-85.

8 Fross RD, Martin WR, Li D, et al. Lesions of the putamen: Their relevance to dystonia. Neurology 1987;37: 1125-9.

9 Mao CC, Gancher ST, Herndon RM. Movement disorders in multiple sclerosis. Mov Disord 1988;3: disorde

10 Coleman RJ, Quinn NP, Marsden CD. Multiple sclerosis presenting as adult onset dystonia. Mov Disord 1988; 3:329-32. 